\title{
Evaluating the role of kangaroo mother care on the lactation among the newborn low birth weight babies
}

\author{
Purnima Margekar1*, Premlata Parekh', Shubha Laxmi Margekar²
}

\begin{abstract}
${ }^{1}$ Department of Paediatrics, Sri Aurobindo Institute of Medical Sciences, Indore, Madhya Pradesh, India
${ }^{2}$ Department of Medicine, Lady Hardinge Medical College, New Delhi, India
\end{abstract}

Received: 22 November 2020

Accepted: 04 January 2021

\section{*Correspondence:}

Dr. Purnima Margekar,

E-mail: dr_purnima2010@yahoo.com

Copyright: (c) the author(s), publisher and licensee Medip Academy. This is an open-access article distributed under the terms of the Creative Commons Attribution Non-Commercial License, which permits unrestricted non-commercial use, distribution, and reproduction in any medium, provided the original work is properly cited.

\begin{abstract}
Background: A universally available and biologically sound method of care for all new-borns, but in particular for premature babies, with three components including skin-to-skin contact, exclusive breastfeeding and support to the "mother infant dyad". The present study was done to evaluate the role of kangaroo mother care (KMC) on the lactation.

Methods: A prospective case control study of KMC was conducted in a teaching institution with a tertiary level neonatal intensive care unit (NICU) over a 12-month period from August 2013 to August 2014 on 50 new-borns weighing less than $1.8 \mathrm{~kg}$. In order to provide $\mathrm{KMC}$, special bag or kangaroo pouch was designed to keep the baby in close contact with mother. The primary outcome variable was "breast feeding". Mode of feeding at admission to either group was noted down. Before allocating to either group, it was assured that baby was tolerating enteral feeds $\&$ there was no regurgitation.

Results: Sixty six percent of neonates in case group and eighty percent of control group were on tube feed while $30 \%$ of case group and $16 \%$ of control group were on spoon feed at the time of enrolment in study. Spoon feeding plus breast feeding was established $28 \%$ of KMC group and in $4 \%$ control group, while only breast feeding was established in $30 \%$ of KMC group and $26 \%$ of control group on discharge.

Conclusions: More babies in KMC group were shifted to breast-feeding on discharge. In addition to that KMC group demonstrated more weight gain and duration of stay was shorter in them.
\end{abstract}

Keywords: Breastfeeding, Lactation, Neonatal intensive care unit, New born

\section{INTRODUCTION}

Maternal-infant skin to skin contact, Kangaroo Mother Care (KMC) was initiated by Rey and Martinez for low birth weight infants in 1979. One third of babies delivered in developing countries like India are low birth weight (LBW). Prolonged medical care is important for many small preterm infants. However, KMC is an effective way to meet baby's needs for warmth, breastfeeding, protection from infection, stimulation, safety and love. Using KMC is cost- effective, most feasible, readily available and has abundant advantages for mother and infant. ${ }^{1}$
A universally available and biologically sound method of care for all new-borns, but in particular for premature babies, with three components which include skin-to-skin Contact, exclusive breastfeeding and support by the "mother infant dyad". 2 In KMC, the mother acts as an incubator through skin-to-skin contact and this enables the baby to be discharged earlier, with fewer complications and the mother can continue to care for her baby at home. ${ }^{3}$ Skin to skin contact stimulates breast milk production so baby gets all the benefits of breast milk. ${ }^{2}$ Despite the said advantages of KMC, it is still not a widely practiced method of care of LBW infants in India. 
The present study was done to evaluate the role of $\mathrm{KMC}$ on the lactation.

\section{METHODS}

TA prospective case control study of Kangaroo Mother Care was conducted in a teaching institution with a tertiary level neonatal intensive care unit (NICU) over a 12-month period from August 2013 to August 2014 on 50 new-borns weighing less than $1.8 \mathrm{~kg}$. An equal number of new-borns from the same setting, matched for weight and gestational age received routine care (warmer), and acted as controls.

In order to provide KMC, special bag or kangaroo pouch was designed to keep the baby in close contact with mother. The primary outcome variable was "breast feeding".

All the new-borns less than $1.8 \mathrm{~kg}$ regardless of their gestational age who were otherwise healthy or had recovered from their illness and the mothers who were willing were included in the case group in this study. Sick unstable newborns, newborn needing ventilator care, newborn with major congenital malformation and weight greater than $1.8 \mathrm{~kg}$ were excluded from the study.

Thorough examination was done before allocating the newborns to either group. The following points were stressed upon: Peripheral pulses and murmur, CRT (Capillary Refill time), skin for icterus and cyanosis, sensorium, AF (Anterior fontanelle), neonatal reflexes, congenital malformation, birth trauma, sclerema.

Mode of feeding at admission to either group was noted down. i.e. whether on tube feed, spoon-feed or breastfed. Before allocating to either group, it was assured that baby was tolerating enteral feeds and there was no regurgitation. The mothers were motivated for KMC. KMC rules were explained. Mother who was ready to do KMC was allocated to KMC group and those who refused were allocated to the control group. Randomization was not possible due to lack of resources and institutional problems.

Through scheduled conversation, mothers were always reminded about the importance and benefits of breastfeeding the baby. The infant with weak sucking were fed with EBM (expressed breast milk) via spoon-feed or tube-feed, until he is capable of emptying at least one breast during the feeding. If the infant gets easily tired while sucking and/or gaining weight less than $10 \mathrm{~g}$ per day it will be explained to the mother the manual expression technique to alternate the lactation with expressed breast milk by spoon feed or tube feed. In the $\mathrm{KMC}$, there was no limit of frequency for breastfeeds. On contrary, more frequent the mother is able to do it; the more benefits will come from it. We motivated the mother regularly about the benefits of breastfeeding. Statistical analysis was done using, mean, standard deviation, error of difference between two means and Student paired ' $\mathrm{t}$ ' test to test the significance ( $\mathrm{P}$ value) between the two groups.

\section{RESULTS}

In both group $94 \%$ babies were delivered by vaginal route and $6 \%$ by LSCS. In case group $44 \%$ were male and $56 \%$ were female while in control group $58 \%$ were male and $42 \%$ were female.

The following chart shows the mode of feeding on admission. On admission, the proportion of new-born baby having breast-feeding was same in both the groups.

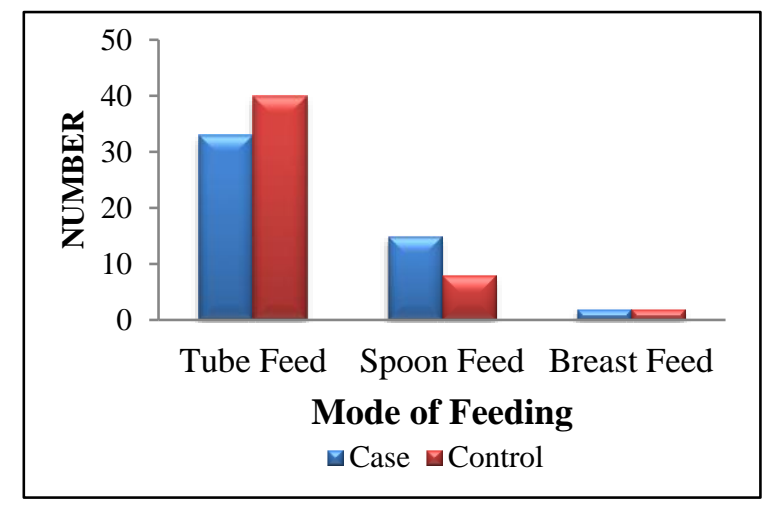

Figure 1: Mode of feeding on admission.

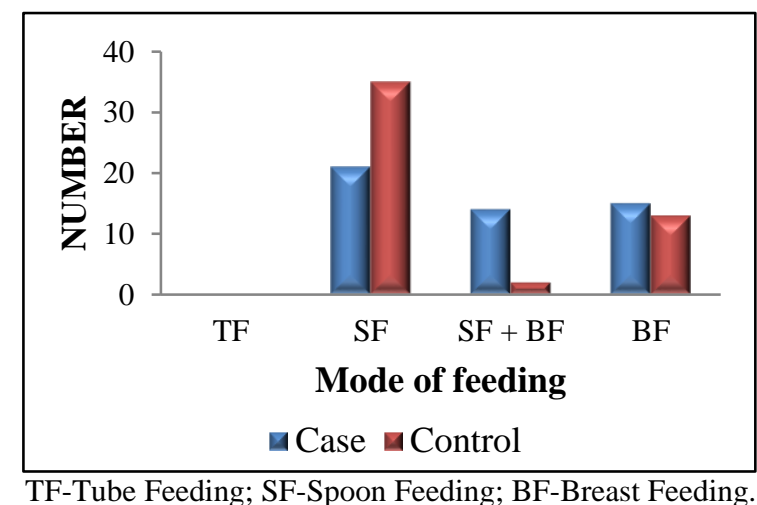

Figure 2: Mode of feeding at discharge

\section{DISCUSSION}

LBW/ preterm babies are associated with high neonatal/infant mortality and morbidity. Of the estimated 4 million neonatal deaths, LBW/preterm infants accounts for more than $33 \%$, hypothermia and infections are frequent aggravating factors for poor outcome of premature/LBW babies. Incubators, open care systems, warmers used in conventional care are expensive and their maintenance and repair are difficult. ${ }^{4}$

Kangaroo mother care is an effective way to meet baby's needs for warmth, growth, well-being, breast feeding, protection from infection, stimulation, safety and love. ., $^{\circ}$ 
Hence, this study was done to assess the effect and benefits of Kangaroo mother care on lactation in low birth weight babies.

Low birth weight babies frequently show an exaggerated weight loss and slower weight gain in the postnatal period due to inadequate caloric intake, frequent illness, hypothermia and occult sepsis. ${ }^{7}$ Therefore, it requires Kangaroo Mother Care for a better feeding and a weight gain, which can be seen in our study.

In our study maximum number of neonates i.e. $66 \%$ KMC group and $80 \%$ control group were on tube feeding at the time of enrollment in study. Breast feeding was established in $30 \%$ in $\mathrm{KMC}$ group and in $26 \%$ control group on discharge ( $\mathrm{p}$ value $<0.05)$; while spoon feeding along with breast feeding was established in $28 \%$ in KMC group and in $4 \%$ control group on discharge.

Ali et al stated that exclusive breast feeding was found to be more prevalent in the KMC group as compared to the control group. Proportions of infants who were exclusively breastfed were higher at 40 weeks (KMC: 94.4\%, control: $72.0 \% \mathrm{p}=0.002$ ); 3 months (KMC: 89.6\%, control: $62.2 \% \mathrm{p}=0.002)$; 6 months postconceptional age (KMC: $84.6 \%$, control: $55.5 \%$, $\mathrm{p}=0.006$ ) in the KMC group, the difference being statistically significant. ${ }^{8}$ Suman et al showed that more KMC babies were exclusively breastfed at the end of the study $(98 \%$ vs. $76 \%){ }^{9}$

Gathwala et al demonstrated that the exclusive breastfeeding rate at end of three months was $88 \%$ in the KMC group compared to $72 \%$ in the control group $(\mathrm{p}<0.05){ }^{10}$ There was no death in KMC group. This may be due to the selection of only stable babies.

Many fallacies were overcome about the doubts and difficulties of initiating, implementing $\mathrm{KMC}$ in a limited resource setting which caters to a population with rural background and low literacy rates.

\section{CONCLUSION}

We concluded that more babies were shifted to breastfeeding on discharge in KMC group. The babies in KMC group also demonstrated more weight gain and had shorter duration of stay. Therefore, effect of KMC on daily weight gain and duration of stay were statistically significant.

\section{Funding: No funding sources}

Conflict of interest: None declared

Ethical approval: The study was approved by the Institutional Ethics Committee

\section{REFERENCES}

1. Kirsten GF, Bergman NJ, Hann FM. Kangaroo mother care in the nursery. Pediatr Clin North Am. 2001;48(2):443-52.

2. Charpak N, Ruiz-Peláez JG. Figueroa de Calume Z. Current knowledge of Kangaroo mother intervention. Curr Opin Pediatr. 1996;8(2):108-12.

3. Ludington HS. Kangaroo care: the beast you can do to help your preterm infant. Tehran. Vista Publication; 2009.

4. Tafari N, Olsson EE. Neonatal cold injury in the tropics. Ethiop Med J. 1973;11:57-65.

5. Anderson GC. Current knowledge about skinto-skin (kangaroo) care for preterm infants. J Perinatol. 1991;11:216-26.

6. Tessier R, Cristo M, Velez S. Kangaroo mother care and the bonding hypothesis. Pediatrics. 1998;102:e17.

7. Tully KP, Holditch DD, Whitetraut RC, David R, O'Shea TM, Geraldo V. A test of kangaroo care on preterm infant breastfeeding. J Obstet Gynecol Neonatal Nurs. 2016;45(1):45-61.

8. Ali SM, Sharma J, Sharma R, Alam S. Kangaroo mother care as compared to conventional care for low birth weight babies. Dicle Medical Journal. 2009;36(3):155-60.

9. Suman Rao P, Udani R, Nanavati R. Kangaroo mother care for lowbirth weight infants: a randomized controlled trial. Indian Pediatrics. 2008;45(1):17-23.

10. Gathwala G, Singh B, Balhara B. KMC facilitates mother baby attachment in low birth weight infants. Indian J Pediatrics. 2008;75(1):43-7.

Cite this article as: Margekar P, Parekh P, Margekar SL. Evaluating the role of kangaroo mother care on the lactation among the newborn low birth weight babies. Int J Reprod Contracept Obstet Gynecol 2021;10:579-81. 